\title{
ALGEBRAIC CONSERVATIVE PETRI NETS BASED ON SYMMETRIC GROUPS
}

\author{
Rajeswari . $\mathbf{R}^{1}$, and Thirusangu . $\mathbf{K}^{2}$ \\ ${ }^{1}$ Department of Mathematics, Sathyabama University, Jeppiaar Nagar, Rajiv Gandhi Road, Chennai, India \\ ${ }^{2}$ Department of Mathematics, Anna University, Chennai, India \\ E-mail : 'thangavelraj@gmail.com, ${ }^{2}$ ktsangu@yahoo.com
}

\begin{abstract}
In this paper we define a new sub class of Petri nets called algebraic conservative Petri nets (ACPN) for a given symmetric group $S_{n}$. We prove that the resulting Petri net (ACPN) is a marked graph. In particular, we show that the algebraic conservative Petri nets associated with S3 and S5 has decompositions $\pi=\left\{\pi_{1}, \Pi_{2}, \Pi_{3}, \Pi_{4}, \Pi_{5}\right\}$ and $\pi^{\prime}=\left\{\pi_{1}, \pi_{2}, \Pi_{3}, \Pi_{4}, \ldots, \Pi_{84}\right\}$ respectively, for the sets of places such that each block $\pi_{i}$ is both siphon and trap and hence the underlying directed graphs of these algebraic conservative Petri nets are Eulerian. Also we show that each of the ACPN associated with these groups has a subset of places which are both siphon and trap such that the input transitions equal the output transitions and both of them equal to the set of all transitions of these algebraic conservative Petri nets and hence that the underlying directed graphs of these algebraic conservative Petri nets associated with $\mathrm{S}_{3}$ and $\mathrm{S}_{5}$ are Hamiltonian.
\end{abstract}

Keywords: Algebraic Conservative Petri Nets, Siphons, Traps, Symmetric Groups, Directed Graphs

\section{INTRODUCTION}

Petri net is a mathematical modeling tool for concurrent systems and has been widely investigated by many researchers [1,2]. A Petri net consists of two kinds of nodes called places and transitions. Directed arcs are used to connect places to transitions and transitions to places. Small dots, called tokens in the places represent a marking of a Petri net. In a graphical representation of a Petri net the places are represented by circles and transitions are represented by bars or small boxes. Various areas of applications of Petri nets include modeling and analysis of the distributed systems, parallel processes, information systems, databases, communication protocols,

The study of structural properties and behavioral properties for marked graphs has been made utilizing siphons and traps $[4,5,6]$. A nonempty subset of places $J$ is called a siphon if every transition having an output place in $J$ has an input place in $J$. A nonempty subset of places $Q$ is called a trap if every transition having an input place in $Q$ has an output place in $Q$.

In this paper we define a new sub class of Petri nets called algebraic conservative Petri nets $(A C P N)$ for a given symmetric group $S_{n}$. We prove that the resulting Petri net (ACPN) is a marked graph . In particular, for the groups $S_{3}$ and $S_{5}$, we show that each of the ACPN associated with computer hardware architectures, manufacturing systems, formal languages and automata, learning theory and graph theory $[5,7]$.

These groups has a subset of places which are both siphon and trap such that the input transitions equal the output transitions and both of them equal to the set of all transitions of these algebraic conservative Petri nets and hence that the underlying directed graphs of these algebraic conservative Petri nets are Hamiltonian. Also we show that the algebraic conservative Petri nets associated with $S_{3}$ and $S_{5}$ has decompositions $\pi$ $=\left\{\pi_{1}, \Pi_{2}, \Pi_{3}, \Pi_{4}, \Pi_{5}\right\}$ and $\Pi^{\prime}=\left\{\pi_{1}, \Pi_{2}, \Pi_{3}, \Pi_{4}, \ldots, \Pi_{84}\right\}$ respectively, for the sets of places such that each block , is both siphon and trap and hence the underlying directed graphs of these algebraic conservative Petri nets associated with $S_{3}$ and $S_{5}$ are Eulerian.

\section{PRELIMINARIES}

In this section we present some basic definitions relevant to this paper.

Definition 2.1: APetri net is triple $N=(P, T, F)$ where $P$ is a finite set of places, $T$ is finite set of transitions such that

(I) $P \cup T \neq ; P \cap=\varphi$

(ii) $O F \subseteq(P \times T) \cup(T \times P)$ is set of directed arcs.

Forall $p P^{*} p=\{t \in T \mid(t, p) \in F\}$ and $p^{*}=\{t \in T \mid(p, t$ )$\in F\}$ be the input and output sets of $p$ respectively. Similarly for all $t \in T,{ }^{*} t=\{p \in P \mid(p, t) \in F\}$ and $t^{\bullet}=\{p \in$ $P \mid(t, p) \in F\}$ be the input and output sets of $t$ respectively.

Definition 2.2: A Petri net is said to be a marked graph if $p|=| p \mid=1$ for all $p \in P$.

Definition 2.3: A Petri net is said to be conservative if $\mid{ }^{\circ} t$ $=\left|t^{\bullet}\right|$ for all $t \in T$.

Definition 2.4: A non-empty subset of places $J$ in a Petri net is called a siphon if ${ }^{\prime} J \subseteq J^{*}$. That is every transition having an output place in $J$ has an input place in $J$. 
Definition 2.5: A nonempty subset of places $Q$ in a Petri net graph is called a trap if $Q^{*} \subseteq Q^{*}$. That is every transition having an input place in $Q$ has an outputplace in $Q$.

Definition 2.6: A non empty subset $Z$ of places in a Petri net graph is said to be both siphon and trap if ${ }^{\circ} Z=Z^{\circ}$. That is, every transition having an input place in $Z$ has an output place in $Z$ and vice versa.

Example 2.7: Consider a Petri net shown in Fig. 1

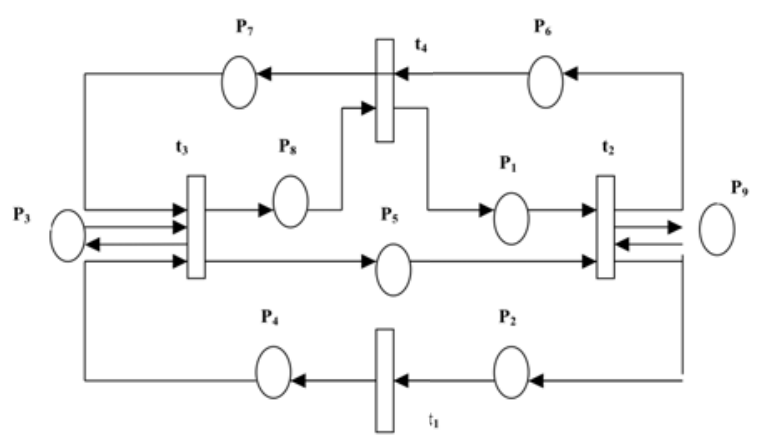

Fig .1 A Petri net

In this Petrinet let $J=\left\{p_{1}, p_{6}, p_{7}\right\}$. Then $J=\left\{t_{2}, t_{4}\right\}$, and $J^{\bullet}=$ $\left\{t_{2}, t_{3}, t_{4}\right\}$. Here $\cdot J \subseteq J^{*}$. Therefore $J$ is a siphon. Let $Q=$ $\left\{p_{6}, p_{7}, p_{8}\right\}$. Then ${ }^{\circ} Q=\left\{t_{2}, t_{3}, t_{4}\right\}, Q^{*}=\left\{t_{3}, t_{4}\right\}$. Here $Q^{*} \subseteq{ }^{*}$ $Q$. Therefore $Q$ is a trap.

Let $Z=\left\{p_{2}, p_{4}, p_{5}\right\}$. Now, $Z^{\prime}=\left\{t_{1}, t_{2}, t_{3}\right\}, Z^{*}=\left\{t_{1}, t_{2}, t_{3}\right\}$. Here $Z^{\bullet}={ }^{\prime} Z$. Therefore $Z$ is both siphon and trap.

Definition 2.8: The symmetric group $S_{n}$ is the group of all permutations on $n$ symbols. This $S_{n}$ is called a permutation group of order $n$ !

Example 2.9: Consider the group $S_{3}=\{\alpha, \beta, \gamma, \delta, \varphi, \psi\}$ where $\alpha=(1)$ (2) (3), $\beta=(13)(2), \gamma=(12)(3), \quad \delta=(132), \varphi$ $=(1)(23), \Psi=(123)$ with generating set $S=\{\alpha, \Psi\}$

\section{ALGEBRAIC CONSERVATIVE PETRI NETS}

In this section we define the new sub class of Petri nets and prove the main results.

Theorem 3.1: There exist a algebraic conservative Petri net for every symmetric group with a generating set.

Proof: Let $S_{n}$ be a symmetric group with generating set $S$ $=\{(1,2),(1,2,3, \ldots, n)\}$. Let $\gamma=(1,2)$ and $\delta=(1,2,3, \ldots, n)$. Take the elements $t_{1}, t_{2}, t_{3}, t_{4}, \ldots . ., t_{n !}$ of the group $S_{n}$ as transitions and set $T=\left\{t_{1}, t_{2}, t_{3}, t_{4}, \ldots ., t_{n !}\right\}$. Now $S \subseteq T$. For every $t_{i} \in T$ and $t_{k} \in S$ such that $t_{i} t_{k}=t_{j}$ introduce a place $p$ such that $p=t_{i}$ and $p^{*}=t_{j}$. Since the generating set $S$ has 2 elements and the group has $n$ ! elements, this process will yield a Petri net $N$ with place set $P$ and the transition set $T$ such that $|T|=n !$ and $\mid P$
I = 2. $n !$. Since the set $S$ has exactly two elements, we have that each transition has exactly two input places and exactly two output places. Now keep tokens in a place $p$ if $p$ is the input of $t_{i}, t_{j}$ for every $t_{i}, t_{j} \in S$. Thus we have constructed a conservative Petri net with initial marking. The resulting Petri net on $S_{n}$ with generating set as $S$ is called algebraic conservative Petri net denoted as $\operatorname{ACPN}\left(S: S_{n}\right)$.

Proposition 3.2: Every algebraic conservative Petri net ( ACPN ) for the symmetric group of order $n$ !, with a generating set is a marked graph.

Proof: From the construction of algebraic conservative Petri net, ( Theorem 3.1) the places are introduced in such way that each place has exactly one input transition and exactly one output transition. Hence by definition, it is a marked graph.

Lemma 3.3 The algebraic conservative Petri net on $S_{n}$ is bounded but not 1-safe.

Proof: From the construction of algebraic conservative Petri net, a token is deposited in a place $p$ if $p$ is the input of $t_{i}, t_{j}$ for every $t_{i}, t_{j} \in S$. So, initially $n$ ! places will receive tokens. Since the this Petri net is conservative, the tokens deposited in this net is neither created nor destroyed. Hence any place can have a maximum of $n$ ! tokens and thus it is bounded. Again, there is a possibility of a place which can have $n$ ! tokens in it, we conclude that it is not 1-safe.

The construction of underlying directed graph $\mathrm{Ns}_{n}$ for the given marked graph $N$ is given in $[4,5]$.

Theorem 3.4: If the $\operatorname{ACPN}\left(S: S_{n}\right)$ for a given symmetric group $S_{n}$ has a subset of places $Z$ such that ' $Z=Z$ ' $=T$ where $T$ is the set of all transitions of $\operatorname{ACPN}\left(S: S_{n}\right)$, then the underlying directed graph $N s_{n}$ has a Hamiltonian circuit.

Proof: In [4] it is proved that if there exist a subset $Z$ of places in a marked graph such that ' $Z=Z^{\prime}=T$, where $T$ is the set of all transitions of the marked graph then the edges corresponding to the places in $Z$ constitute a directed Hamiltonian circuit in the underlying directed graph. Hence by proposition 3.2, the theorem follows.

Theorem 3.5: If the place set $P$ of $\operatorname{ACPN}\left(S: S_{n}\right)$ for a given symmetric group $S_{n}$ has a decomposition $\pi=\left\{\pi_{1}, \pi_{2}\right.$, $\left.\pi_{3}, \ldots, \pi_{n}\right\}$ in which each block ${ }_{\mathrm{i}}$ both siphon and trap then the underlying directed graph $N s_{n}$ of $\operatorname{ACPN}\left(S: S_{n}\right)$ is Eulerian.

Proof: $\ln [5]$ it is proved that if there exist a decomposition $\pi=\left\{\pi_{1}, \pi_{2}, \pi_{3}, \ldots, \pi_{n}\right\}$ for the set of places of a marked 
graph such that each block ${ }_{i}$ in the decomposition $\pi=$ $\left\{\pi_{1}, \pi_{2}, \pi_{3}, \ldots, \pi_{n}\right\}$ is both siphon and trap then the underlying directed graph of that marked graph is Eulerian. Hence by proposition 3.2, the theorem follows.

Example 3.6: Consider the symmetric group given in example 2.9. The algebraic conservative Petri net for the group $S_{3}, A C P N\left(S: S_{3}\right)$ is shown in Fig.2. Clearly this is a marked graph.

Let $\pi=\left\{\pi_{1}, \pi_{2}, \pi_{3}, \pi_{4}, \pi_{5}\right\}$ be the partition of the set of places of $S_{3}$, where $\pi_{1}=\left\{p_{2}, p_{3}\right\}$,

$\pi_{2}=\left\{p_{5}, p_{6}, p_{7}\right\}, \pi_{3}=\left\{p_{8}, p_{9}\right\}, \pi_{4}=\left\{p_{10}, p_{11}\right\}, \pi_{5}=\left\{p_{1}, p_{4}, p_{12}\right\}$ Such that ${ }^{\circ} \pi_{1}=\{\alpha, \lambda\}$,

${ }^{\cdot} \pi_{1}=\{\alpha, \lambda\}$. That is, $\dot{\pi}_{1}=\pi_{1}^{\cdot}$. Therefore ${ }_{1}$ is both siphon and trap.

Similarly, $\pi_{2}=\{\beta, \varphi, \lambda\}, \pi_{2}^{*}=\{\beta, \varphi, \lambda\}$. That is, ${ }^{\circ} \pi_{2}$ $=\pi_{2}{ }^{\circ}$. Therefore ${ }^{\circ} \pi_{2}$ is both siphon and trap. ${ }^{\circ} \pi_{3}=\{\beta, \sigma\}$, $\pi_{3}{ }^{\circ}=\{\beta, \sigma\}$. That is, $\quad \pi_{3}=\pi_{3}{ }^{\circ}$. Therefore ${ }_{3}$ is both siphon and trap. ${ }^{\circ} \pi_{4}=\{\boldsymbol{\varphi}, \boldsymbol{\Psi}\}, \pi_{4}{ }^{\circ}=\{\boldsymbol{\varphi}, \boldsymbol{\Psi}\}$. That is, $\pi_{4}=\pi_{4}^{\cdot}$. Therefore ${ }_{4}$ is both siphon and trap.

$\pi_{5}=\{\boldsymbol{\alpha}, \boldsymbol{\Psi}, \boldsymbol{\delta}\}, \quad \pi_{5} \cdot \mathbf{a}=\{\boldsymbol{\alpha}, \boldsymbol{\Psi}, \boldsymbol{\delta}\}$, That is, $\pi_{5}=\pi_{5} \cdot$ Therefore $\pi_{5}$ is both siphon and trap. Hence the underlying directed graph of the marked graph for $S_{3}$ is Eulerian.

Again in $\operatorname{ACPN}\left(S: S_{3}\right)$ there exist a subset $Z=\left\{p_{2}, p_{6}, p_{7}\right.$, $\left.p_{8}, p_{12}, p_{4}\right\}$ such that $Z=Z^{\prime}=T$. Therefore in the underlying directed graph, the edges $\left\{e_{1}, e_{3}, e_{4}, e_{6}, e_{10}, e_{11}\right\}$ corresponding to the places in $Z$ constitutes a directed Hamiltonian circuit.



Fig .2 The algebraic conservative Petri net for the group given in example 2.9

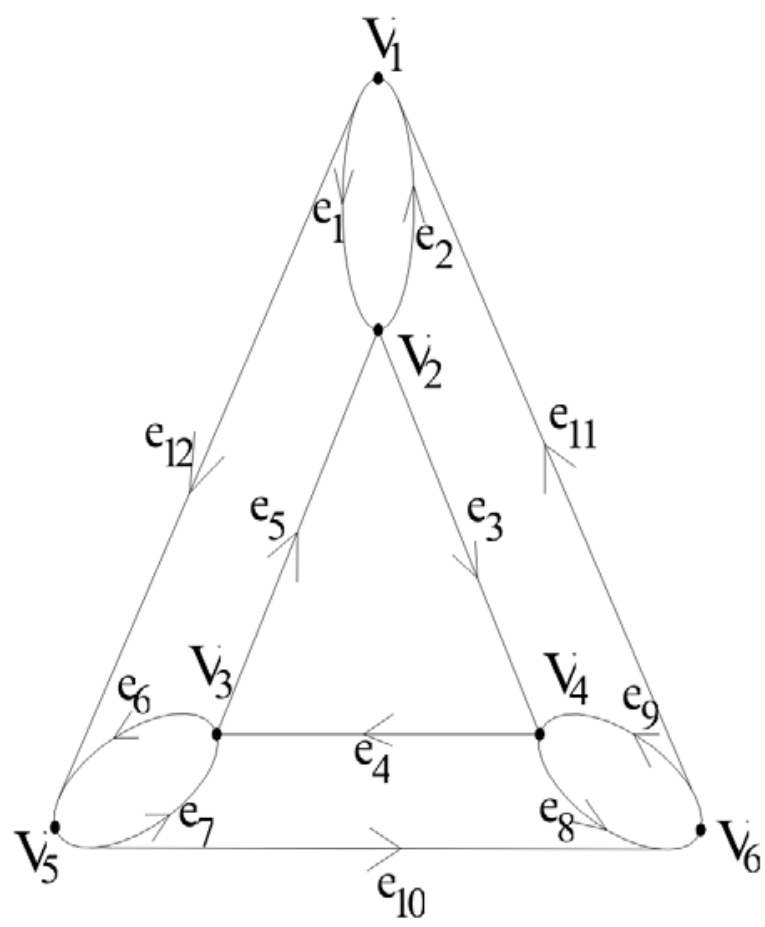

Fig .3 The underlying directed graph for the algebraic conservative Petri net for the Fig .2

Example 3.7: Consider the symmetric group $S_{5}$, consisting of 120 elements as follows.

\begin{tabular}{|l|l|l|l|l|}
\hline 12345 & 21345 & 31245 & 41235 & 51234 \\
\hline 12354 & 21354 & 31254 & 41253 & 51243 \\
\hline 12534 & 21534 & 31425 & 41523 & 51342 \\
\hline 12543 & 21543 & 31452 & 41532 & 51324 \\
\hline 12435 & 21453 & 31524 & 41325 & 51423 \\
\hline 12453 & 21435 & 31542 & 41352 & 51432 \\
\hline 13245 & 23145 & 32145 & 42135 & 52134 \\
\hline 13254 & 23154 & 32154 & 42153 & 52143 \\
\hline 13425 & 23415 & 32514 & 42513 & 52314 \\
\hline 13452 & 23451 & 32541 & 42531 & 52341 \\
\hline 13524 & 23514 & 32415 & 42315 & 52413 \\
\hline 13542 & 23541 & 32451 & 42351 & 52431 \\
\hline 14235 & 24315 & 34251 & 43251 & 53421 \\
\hline 14253 & 24351 & 34215 & 43215 & 53412 \\
\hline 14325 & 24531 & 34521 & 43521 & 53241 \\
\hline 14352 & 24513 & 34512 & 43512 & 53214 \\
\hline 14523 & 24135 & 34125 & 43125 & 53142 \\
\hline 14532 & 24513 & 34152 & 43152 & 53124 \\
\hline 15432 & 25413 & 35142 & 45132 & 54321 \\
\hline 15423 & 25431 & 35124 & 45123 & 54312 \\
\hline 15243 & 25341 & 35421 & 45321 & 54213 \\
\hline 15234 & 25314 & 35412 & 45312 & 54231 \\
\hline 15342 & 25143 & 35214 & 45231 & 54123 \\
\hline 15324 & 25134 & 35241 & 45213 & 54132 \\
\hline & & & & \\
\hline
\end{tabular}


Let $S_{5}$ be a symmetric group with generating set $S=\left\{\left(\begin{array}{ll}1 & 2\end{array}\right),(12345)\right\}$. Let $\gamma=\left(\begin{array}{ll}1 & 2\end{array}\right)$ and $\quad \psi=(12345)$. Since $S_{5}$ has 120 elements, take the elements of $S_{5}$ as the transition set of conservative Petri net. That is, $T=\left\{t_{1}, t_{2}, t_{3}, \ldots, t_{12}\right\}$. Based on the theorem, we have 240 places namely $P=\left\{p_{1}, p_{2}, p_{3}, p_{4}, \ldots . ., p_{240}\right\}$. The input and outputs of transitions are given in the following table. This leads to the following conservative Petri net $A C P N\left(S: S_{5}\right)$.

\begin{tabular}{|c|c|c|c|c|}
\hline $\begin{aligned} \mathrm{t}_{1} & =\left\{\mathrm{p}_{2}, \mathrm{p}_{125}\right\} \\
\mathrm{t}_{1} & =\left\{\mathrm{p}_{1}, \mathrm{p}_{121}\right\}\end{aligned}$ & $\begin{aligned} & \mathrm{t}_{2}=\left\{\mathrm{p}_{4}, \mathrm{p}_{130}\right\} \\
& \mathrm{t}_{2} \cdot{ }^{\circ}=\left\{\mathrm{p}_{3}, \mathrm{p}_{126}\right\}\end{aligned}$ & $\begin{aligned} \mathrm{t}_{3} & =\left\{\mathrm{p}_{6}, \mathrm{p}_{135}\right\} \\
\mathrm{t}_{3} \cdot & =\left\{\mathrm{p}_{5}, \mathrm{p}_{131}\right\}\end{aligned}$ & $\begin{aligned}{ }^{\circ} \mathrm{t}_{4} & =\left\{\mathrm{p}_{8}, \mathrm{p}_{140}\right\} \\
\mathrm{t}_{4}{ }^{\circ} & =\left\{\mathrm{P}_{7}, \mathrm{p}_{136}\right\}\end{aligned}$ & $\begin{aligned} & \mathrm{t}_{5}=\left\{\mathrm{p}_{10}, \mathrm{p}_{145}\right\}, \\
& \mathrm{t}_{5} \cdot=\left\{\mathrm{p}_{9}, \mathrm{p}_{141}\right\}\end{aligned}$ \\
\hline $\begin{aligned}{ }^{\circ} \mathrm{t}_{6} & =\left\{\mathrm{p}_{12}, \mathrm{p}_{150}\right\}, \\
\mathrm{t}_{6}{ }^{\circ} & =\left\{\mathrm{p}_{11}, \mathrm{p}_{146}\right\}\end{aligned}$ & $\begin{aligned} & \mathrm{t}_{7}=\left\{\mathrm{p}_{14}, \mathrm{p}_{155}\right\}, \\
& \mathrm{t}_{7} \cdot=\left\{\mathrm{p}_{13}, \mathrm{p}_{151}\right\}\end{aligned}$ & $\begin{aligned} \mathrm{t}_{8} & =\left\{\mathrm{p}_{16}, \mathrm{p}_{160}\right\}, \\
\mathrm{t}_{8} & =\left\{\mathrm{p}_{15}, \mathrm{p}_{156}\right\}\end{aligned}$ & $\begin{aligned} \mathrm{t}_{9} & =\left\{\mathrm{p}_{18}, \mathrm{p}_{165}\right\} \\
\mathrm{t}_{9} & =\left\{\mathrm{p}_{17}, \mathrm{p}_{161}\right\}\end{aligned}$ & $\begin{aligned} \mathrm{t}_{10} & =\left\{\mathrm{p}_{20}, \mathrm{p}_{170}\right\}, \\
\mathrm{t}_{10} & =\left\{\mathrm{P}_{19}, \mathrm{P}_{166}\right\}\end{aligned}$ \\
\hline $\begin{aligned} \mathrm{t}_{11} & =\left\{\mathrm{p}_{22}, \mathrm{p}_{175}\right\}, \\
\mathrm{t}_{11} & =\left\{\mathrm{p}_{21}, \mathrm{p}_{171}\right\}\end{aligned}$ & $\begin{aligned} \cdot \mathrm{t}_{12} & =\left\{\mathrm{p}_{24}, \mathrm{p}_{180}\right\} \\
\mathrm{t}_{12} & =\left\{\mathrm{p}_{23}, \mathrm{p}_{176}\right\}\end{aligned}$ & $\begin{aligned} & \mathrm{t}_{13}=\left\{\mathrm{p}_{26}, \mathrm{p}_{185}\right\} \\
& \mathrm{t}_{13} \cdot=\left\{\mathrm{p}_{25}, \mathrm{p}_{181}\right\}\end{aligned}$ & $\begin{aligned} \mathrm{t}_{14} & =\left\{\mathrm{p}_{28}, \mathrm{p}_{190}\right\}, \\
\mathrm{t}_{14} & =\left\{\mathrm{p}_{27}, \mathrm{p}_{186}\right\}\end{aligned}$ & $\begin{aligned} \mathrm{t}_{15} & =\left\{\mathrm{p}_{30}, \mathrm{p}_{195}\right\} \\
\mathrm{t}_{15} & =\left\{\mathrm{p}_{29}, \mathrm{p}_{191}\right\}\end{aligned}$ \\
\hline $\begin{aligned} \mathrm{t}_{16} & =\left\{\mathrm{p}_{32}, \mathrm{p}_{200}\right\}, \\
\mathrm{t}_{16} & =\left\{\mathrm{p}_{31}, \mathrm{p}_{136}\right\}\end{aligned}$ & $\begin{aligned} \cdot \mathrm{t}_{17} & =\left\{\mathrm{p}_{34}, \mathrm{p}_{205}\right\} \\
\mathrm{t}_{17} & =\left\{\mathrm{p}_{33}, \mathrm{p}_{201}\right\}\end{aligned}$ & $\begin{aligned} \mathrm{t}_{18} & =\left\{\mathrm{p}_{36}, \mathrm{p}_{210}\right\} \\
\mathrm{t}_{18} & =\left\{\mathrm{p}_{35}, \mathrm{p}_{206}\right\}\end{aligned}$ & $\begin{aligned} \mathrm{t}_{19} & =\left\{\mathrm{p}_{38}, \mathrm{p}_{215}\right\} \\
\mathrm{t}_{19} & =\left\{\mathrm{p}_{37}, \mathrm{p}_{211}\right\}\end{aligned}$ & $\begin{aligned} \mathrm{t}_{20} & =\left\{\mathrm{p}_{40}, \mathrm{p}_{220}\right\}, \\
\mathrm{t}_{20} & =\left\{\mathrm{p}_{39}, \mathrm{p}_{216}\right\}\end{aligned}$ \\
\hline $\begin{aligned} \mathrm{t}_{21} & =\left\{\mathrm{p}_{42}, \mathrm{p}_{225}\right\}, \\
\mathrm{t}_{21} & =\left\{\mathrm{p}_{41}, \mathrm{p}_{221}\right\}\end{aligned}$ & $\begin{aligned} \mathrm{t}_{22} & =\left\{\mathrm{p}_{44}, \mathrm{p}_{230}\right\}, \\
\mathrm{t}_{22} & =\left\{\mathrm{p}_{43}, \mathrm{p}_{226}\right\}\end{aligned}$ & $\begin{aligned} & \mathrm{t}_{23}=\left\{\mathrm{p}_{46}, \mathrm{p}_{235}\right\} \\
& \mathrm{t}_{23}{ }^{\circ}=\left\{\mathrm{p}_{45}, \mathrm{p}_{231}\right\}\end{aligned}$ & $\begin{aligned} \mathrm{t}_{24} & =\left\{\mathrm{p}_{48}, \mathrm{p}_{240}\right\}, \\
\mathrm{t}_{24} & =\left\{\mathrm{p}_{47}, \mathrm{p}_{236}\right\}\end{aligned}$ & $\begin{aligned} \mathrm{t}_{25} & =\left\{\mathrm{p}_{1}, \mathrm{p}_{226}\right\} \\
\mathrm{t}_{25} & =\left\{\mathrm{p}_{2}, \mathrm{p}_{227}\right\}\end{aligned}$ \\
\hline $\begin{aligned}{ }^{\circ} \mathrm{t}_{26} & =\left\{\mathrm{p}_{3}, \mathrm{p}_{221}\right\}, \\
\mathrm{t}_{26} & =\left\{\mathrm{p}_{4}, \mathrm{p}_{222}\right\}\end{aligned}$ & $\begin{aligned}{ }^{\circ} \mathrm{t}_{27} & =\left\{\mathrm{p}_{5}, \mathrm{p}_{216}\right\} \\
\mathrm{t}_{27}{ }^{\circ} & =\left\{\mathrm{p}_{6}, \mathrm{p}_{217}\right\}\end{aligned}$ & $\begin{aligned} \mathrm{t}_{28} & =\left\{\mathrm{p}_{7}, \mathrm{p}_{211}\right\} \\
\mathrm{t}_{28} & =\left\{\mathrm{p}_{8}, \mathrm{p}_{212}\right\}\end{aligned}$ & $\begin{aligned}{ }^{\circ} \mathrm{t}_{29} & =\left\{\mathrm{p}_{11}, \mathrm{p}_{231}\right\} \\
\mathrm{t}_{29}{ }^{\circ} & =\left\{\mathrm{p}_{12}, \mathrm{p}_{232}\right\}\end{aligned}$ & $\begin{aligned} \mathrm{t}_{30} & =\left\{\mathrm{p}_{9}, \mathrm{p}_{236}\right\} \\
\mathrm{t}_{30} & =\left\{\mathrm{p}_{10}, \mathrm{p}_{237}\right\}\end{aligned}$ \\
\hline $\begin{aligned}{ }^{\circ} t_{31} & =\left\{p_{13}, p_{131}\right\} \\
t_{31} & =\left\{p_{14}, p_{132}\right\}\end{aligned}$ & $\begin{aligned} \mathrm{t}_{32} & =\left\{\mathrm{p}_{15}, \mathrm{p}_{136}\right\} \\
\mathrm{t}_{32} & =\left\{\mathrm{p}_{16}, \mathrm{p}_{137}\right\}\end{aligned}$ & $\begin{aligned} & \mathrm{t}_{33}=\left\{\mathrm{p}_{17}, \mathrm{p}_{126}\right\} \\
& \mathrm{t}_{33} \cdot=\left\{\mathrm{p}_{18}, \mathrm{p}_{127}\right\}\end{aligned}$ & $\begin{aligned} \mathrm{t}_{34} & =\left\{\mathrm{p}_{19}, \mathrm{p}_{121}\right\} \\
\mathrm{t}_{34} & =\left\{\mathrm{p}_{20}, \mathrm{p}_{122}\right\}\end{aligned}$ & $\begin{aligned} \mathrm{t}_{35} & =\left\{\mathrm{p}_{21}, \mathrm{p}_{146}\right\} \\
\mathrm{t}_{35} & =\left\{\mathrm{p}_{22}, \mathrm{p}_{147}\right\}\end{aligned}$ \\
\hline $\begin{aligned} \mathrm{t}_{36} & =\left\{\mathrm{p}_{23}, \mathrm{p}_{141}\right\} \\
\mathrm{t}_{36}{ }^{\circ} & =\left\{\mathrm{p}_{24}, \mathrm{p}_{142}\right\}\end{aligned}$ & $\begin{aligned} \mathrm{t}_{37} & =\left\{\mathrm{p}_{29}, \mathrm{p}_{156}\right\} \\
\mathrm{t}_{37} & =\left\{\mathrm{p}_{30}, \mathrm{p}_{157}\right\}\end{aligned}$ & $\begin{aligned} \mathrm{t}_{38} & =\left\{\mathrm{p}_{31}, \mathrm{p}_{151}\right\} \\
\mathrm{t}_{38} \cdot & =\left\{\mathrm{p}_{32}, \mathrm{p}_{152}\right\}\end{aligned}$ & $\begin{aligned} \mathrm{t}_{39} & =\left\{\mathrm{p}_{35}, \mathrm{p}_{161}\right\} \\
\mathrm{t}_{39} & =\left\{\mathrm{p}_{36}, \mathrm{p}_{162}\right\}\end{aligned}$ & $\begin{aligned} \mathrm{t}_{40} & =\left\{\mathrm{p}_{33}, \mathrm{p}_{166}\right\} \\
\mathrm{t}_{40} & =\left\{\mathrm{p}_{34}, \mathrm{p}_{167}\right\}\end{aligned}$ \\
\hline $\begin{aligned} \mathrm{t}_{41} & =\left\{\mathrm{p}_{25}, \mathrm{p}_{171}\right\} \\
\mathrm{t}_{41} & =\left\{\mathrm{p}_{26}, \mathrm{p}_{172}\right\}\end{aligned}$ & $\begin{aligned}{ }^{t} t_{42} & =\left\{\mathrm{p}_{27}, \mathrm{p}_{176}\right\} \\
\mathrm{t}_{42} & =\left\{\mathrm{p}_{28}, \mathrm{p}_{177}\right\}\end{aligned}$ & $\begin{aligned} \mathrm{t}_{43} & =\left\{\mathrm{p}_{39}, \mathrm{p}_{196}\right\} \\
\mathrm{t}_{43} \cdot & =\left\{\mathrm{p}_{40}, \mathrm{p}_{197}\right\}\end{aligned}$ & $\begin{aligned}{ }^{\circ} \mathrm{t}_{44} & =\left\{\mathrm{p}_{37}, \mathrm{p}_{191}\right\} \\
\mathrm{t}_{44} & =\left\{\mathrm{p}_{38}, \mathrm{p}_{192}\right\}\end{aligned}$ & $\begin{aligned} \mathrm{t}_{45} & =\left\{\mathrm{p}_{45}, \mathrm{p}_{181}\right\} \\
\mathrm{t}_{45} & =\left\{\mathrm{p}_{46}, \mathrm{p}_{182}\right\}\end{aligned}$ \\
\hline $\begin{aligned} \mathrm{t}_{46} & =\left\{\mathrm{p}_{47}, \mathrm{p}_{186}\right\} \\
\mathrm{t}_{46} & =\left\{\mathrm{p}_{48}, \mathrm{p}_{187}\right\}\end{aligned}$ & $\begin{aligned} \mathrm{t}_{47} & =\left\{\mathrm{p}_{41}, \mathrm{p}_{206}\right\} \\
\mathrm{t}_{47} & =\left\{\mathrm{p}_{42}, \mathrm{p}_{207}\right\}\end{aligned}$ & $\begin{aligned} \mathrm{t}_{48} & =\left\{\mathrm{p}_{43}, \mathrm{p}_{201}\right\} \\
\mathrm{t}_{48} \cdot & =\left\{\mathrm{p}_{44}, \mathrm{p}_{202}\right\}\end{aligned}$ & $\begin{aligned} \mathrm{t}_{49} & =\left\{\mathrm{p}_{50}, \mathrm{p}_{202}\right\} \\
\mathrm{t}_{49} & =\left\{\mathrm{p}_{49}, \mathrm{p}_{203}\right\}\end{aligned}$ & $\begin{aligned} \mathrm{t}_{50} & =\left\{\mathrm{p}_{52}, \mathrm{p}_{207}\right\} \\
\mathrm{t}_{50} & =\left\{\mathrm{p}_{51}, \mathrm{p}_{208}\right\}\end{aligned}$ \\
\hline $\begin{array}{l}\mathrm{t}_{51}=\left\{\mathrm{p}_{54}, \mathrm{p}_{187}\right\} \\
\mathrm{t}_{51}=\left\{\mathrm{p}_{53}, \mathrm{p}_{188}\right\}\end{array}$ & $\begin{aligned} \mathrm{t}_{52} & =\left\{\mathrm{p}_{56}, \mathrm{p}_{182}\right\} \\
\mathrm{t}_{52} & =\left\{\mathrm{p}_{55}, \mathrm{p}_{183}\right\}\end{aligned}$ & $\begin{aligned} \mathrm{t}_{53} & =\left\{\mathrm{p}_{58}, \mathrm{p}_{197}\right\} \\
\mathrm{t}_{53}{ }^{\circ} & =\left\{\mathrm{p}_{57}, \mathrm{p}_{198}\right\}\end{aligned}$ & $\begin{aligned}{ }^{\cdot} \mathrm{t}_{54} & =\left\{\mathrm{p}_{60}, \mathrm{p}_{192}\right\} \\
\mathrm{t}_{54} & =\left\{\mathrm{p}_{59}, \mathrm{p}_{193}\right\}\end{aligned}$ & $\begin{aligned} \mathrm{t}_{55} & =\left\{\mathrm{p}_{49}, \mathrm{p}_{217}\right\} \\
\mathrm{t}_{55} & =\left\{\mathrm{p}_{50}, \mathrm{p}_{218}\right\}\end{aligned}$ \\
\hline $\begin{aligned}{ }^{\circ} t_{56} & =\left\{p_{51}, p_{212}\right\} \\
t_{56} & =\left\{p_{52}, p_{213}\right\}\end{aligned}$ & $\begin{aligned} \mathrm{t}_{57} & =\left\{\mathrm{p}_{57}, \mathrm{p}_{232}\right\} \\
\mathrm{t}_{57} & =\left\{\mathrm{p}_{58}, \mathrm{p}_{233}\right\}\end{aligned}$ & $\begin{aligned} \mathrm{t}_{58} & =\left\{\mathrm{p}_{59}, \mathrm{p}_{237}\right\} \\
\mathrm{t}_{58}{ }^{\circ} & =\left\{\mathrm{p}_{60}, \mathrm{p}_{238}\right\}\end{aligned}$ & $\begin{aligned} \mathrm{t}_{59} & =\left\{\mathrm{p}_{53}, \mathrm{p}_{222}\right\} \\
\mathrm{t}_{59} & =\left\{\mathrm{p}_{54}, \mathrm{p}_{223}\right\}\end{aligned}$ & $\begin{aligned}{ }^{\circ} \mathrm{t}_{60} & =\left\{\mathrm{p}_{55}, \mathrm{p}_{227}\right\} \\
\mathrm{t}_{60} & =\left\{\mathrm{p}_{56}, \mathrm{p}_{228}\right\}\end{aligned}$ \\
\hline $\begin{aligned} \mathrm{t}_{61} & =\left\{\mathrm{p}_{62}, \mathrm{p}_{132}\right\} \\
\mathrm{t}_{61} & =\left\{\mathrm{p}_{61}, \mathrm{p}_{133}\right\}\end{aligned}$ & $\begin{aligned}{ }^{\mathrm{t}} \mathrm{t}_{62} & =\left\{\mathrm{p}_{64}, \mathrm{p}_{137}\right\} \\
\mathrm{t}_{62} & =\left\{\mathrm{p}_{63}, \mathrm{p}_{138}\right\}\end{aligned}$ & $\begin{aligned} & \mathrm{t}_{63}=\left\{\mathrm{p}_{66, \mathrm{p}_{127}}\right\} \\
& \mathrm{t}_{63} \cdot=\left\{\mathrm{p}_{65}, \mathrm{p}_{128}\right\}\end{aligned}$ & $\begin{aligned} \mathrm{t}_{64} & =\left\{\mathrm{p}_{66}, \mathrm{p}_{122}\right\} \\
\mathrm{t}_{64} & =\left\{\mathrm{p}_{65}, \mathrm{p}_{123}\right\}\end{aligned}$ & $\begin{aligned} \mathrm{t}_{65} & =\left\{\mathrm{p}_{63}, \mathrm{p}_{147}\right\} \\
\mathrm{t}_{65} & =\left\{\mathrm{p}_{64}, \mathrm{p}_{148}\right\}\end{aligned}$ \\
\hline $\begin{aligned} \mathrm{t}_{66} & =\left\{\mathrm{p}_{61}, \mathrm{p}_{142}\right\} \\
\mathrm{t}_{66} & =\left\{\mathrm{p}_{62}, \mathrm{p}_{143}\right\}\end{aligned}$ & $\begin{aligned} \mathrm{t}_{67} & =\left\{\mathrm{p}_{68}, \mathrm{p}_{162}\right\} \\
\mathrm{t}_{67} & =\left\{\mathrm{p}_{67}, \mathrm{p}_{163}\right\}\end{aligned}$ & $\begin{aligned} \mathrm{t}_{68} & =\left\{\mathrm{p}_{70}, \mathrm{p}_{167}\right\} \\
\mathrm{t}_{68} & =\left\{\mathrm{p}_{69}, \mathrm{p}_{168}\right\}\end{aligned}$ & $\begin{aligned} \mathrm{t}_{69} & =\left\{\mathrm{p}_{72}, \mathrm{p}_{157}\right\} \\
\mathrm{t}_{69} & =\left\{\mathrm{p}_{71}, \mathrm{p}_{158}\right\}\end{aligned}$ & $\begin{aligned} \mathrm{t}_{70} & =\left\{\mathrm{p}_{71}, \mathrm{p}_{152}\right\} \\
\mathrm{t}_{70} & =\left\{\mathrm{p}_{72}, \mathrm{p}_{153}\right\}\end{aligned}$ \\
\hline $\begin{aligned}{ }^{\circ} t_{71} & =\left\{p_{69}, p_{177}\right\} \\
t_{71} & =\left\{p_{70}, p_{178}\right\}\end{aligned}$ & $\begin{aligned} \mathrm{t}_{72} & =\left\{\mathrm{p}_{67}, \mathrm{p}_{172}\right\} \\
\mathrm{t}_{72} & =\left\{\mathrm{p}_{68}, \mathrm{p}_{173}\right\}\end{aligned}$ & $\begin{aligned} \mathrm{t}_{73} & =\left\{\mathrm{p}_{74}, \mathrm{p}_{168}\right\} \\
\mathrm{t}_{73} \cdot & =\left\{\mathrm{p}_{73}, \mathrm{p}_{169}\right\}\end{aligned}$ & $\begin{aligned} \mathrm{t}_{74} & =\left\{\mathrm{p}_{76,}, \mathrm{p}_{163}\right\} \\
\mathrm{t}_{74} & =\left\{\mathrm{p}_{75}, \mathrm{p}_{164}\right\}\end{aligned}$ & $\begin{aligned} \mathrm{t}_{75} & =\left\{\mathrm{p}_{78}, \mathrm{p}_{153}\right\} \\
\mathrm{t}_{75} & =\left\{\mathrm{p}_{77}, \mathrm{p}_{154}\right\}\end{aligned}$ \\
\hline $\begin{aligned} \mathrm{t}_{76} & =\left\{\mathrm{p}_{80}, \mathrm{p}_{158}\right\} \\
\mathrm{t}_{76} & =\left\{\mathrm{p}_{79}, \mathrm{p}_{159}\right\}\end{aligned}$ & $\begin{aligned} \mathrm{t}_{77} & =\left\{\mathrm{p}_{82}, \mathrm{p}_{178}\right\} \\
\mathrm{t}_{77} & =\left\{\mathrm{p}_{81}, \mathrm{p}_{179}\right\}\end{aligned}$ & $\begin{aligned} \mathrm{t}_{78} & =\left\{\mathrm{p}_{84}, \mathrm{p}_{173}\right\} \\
\mathrm{t}_{78} & =\left\{\mathrm{p}_{83}, \mathrm{p}_{174}\right\}\end{aligned}$ & $\begin{aligned} \mathrm{t}_{79} & =\left\{\mathrm{p}_{73}, \mathrm{p}_{198}\right\} \\
\mathrm{t}_{79} & =\left\{\mathrm{p}_{74}, \mathrm{p}_{199}\right\}\end{aligned}$ & $\begin{aligned} \mathrm{t}_{80} & =\left\{\mathrm{p}_{75}, \mathrm{p}_{193}\right\} \\
\mathrm{t}_{80} & =\left\{\mathrm{p}_{76}, \mathrm{p}_{194}\right\}\end{aligned}$ \\
\hline $\begin{aligned}{ }^{\circ} t_{81} & =\left\{p_{77}, p_{183}\right\} \\
t_{81} & =\left\{p_{78}, p_{184}\right\}\end{aligned}$ & $\begin{aligned} \mathrm{t}_{82} & =\left\{\mathrm{p}_{79}, \mathrm{p}_{188}\right\} \\
\mathrm{t}_{82} & =\left\{\mathrm{p}_{80}, \mathrm{p}_{189}\right\}\end{aligned}$ & $\begin{aligned}{ }^{\cdot} \mathrm{t}_{83} & =\left\{\mathrm{p}_{81}, \mathrm{p}_{208}\right\} \\
\mathrm{t}_{83}{ }^{\circ} & =\left\{\mathrm{p}_{82}, \mathrm{p}_{209}\right\}\end{aligned}$ & $\begin{aligned}{ }^{\circ} t_{84} & =\left\{p_{83}, p_{203}\right\} \\
t_{84} & =\left\{p_{84}, p_{204}\right\}\end{aligned}$ & $\begin{aligned} \mathrm{t}_{85} & =\left\{\mathrm{p}_{86}, \mathrm{p}_{218}\right\} \\
\mathrm{t}_{85} & =\left\{\mathrm{p}_{85}, \mathrm{p}_{219}\right\}\end{aligned}$ \\
\hline $\begin{aligned} \mathrm{t}_{86} & =\left\{\mathrm{p}_{88}, \mathrm{p}_{213}\right\} \\
\mathrm{t}_{86} & =\left\{\mathrm{p}_{87}, \mathrm{p}_{214}\right\}\end{aligned}$ & $\begin{aligned} \mathrm{t}_{87} & =\left\{\mathrm{p}_{90}, \mathrm{p}_{223}\right\} \\
\mathrm{t}_{87} & =\left\{\mathrm{p}_{89}, \mathrm{p}_{224}\right\}\end{aligned}$ & $\begin{aligned} \mathrm{t}_{88} & =\left\{\mathrm{p}_{89}, \mathrm{p}_{228}\right\} \\
\mathrm{t}_{88} & =\left\{\mathrm{p}_{90}, \mathrm{p}_{229}\right\}\end{aligned}$ & $\begin{aligned}{ }^{\circ} t_{89} & =\left\{\mathrm{p}_{87}, \mathrm{p}_{233}\right\} \\
\mathrm{t}_{89} & =\left\{\mathrm{p}_{88}, \mathrm{p}_{234}\right\}\end{aligned}$ & $\begin{aligned}{ }^{t} \mathrm{t}_{90} & =\left\{\mathrm{p}_{85}, \mathrm{p}_{238}\right\} \\
\mathrm{t}_{90} & =\left\{\mathrm{p}_{86}, \mathrm{p}_{239}\right\}\end{aligned}$ \\
\hline $\begin{aligned} \mathrm{t}_{91} & =\left\{\mathrm{p}_{92}, \mathrm{p}_{128}\right\} \\
\mathrm{t}_{91} & =\left\{\mathrm{p}_{91}, \mathrm{p}_{129}\right\}\end{aligned}$ & $\begin{aligned}{ }^{\circ} t_{92} & =\left\{p_{94}, p_{123}\right\} \\
t_{92} & =\left\{p_{93}, p_{124}\right\}\end{aligned}$ & $\begin{aligned} \mathrm{t}_{93} & =\left\{\mathrm{p}_{96}, \mathrm{p}_{138}\right\} \\
\mathrm{t}_{93} & =\left\{\mathrm{p}_{95}, \mathrm{p}_{139}\right\}\end{aligned}$ & $\begin{aligned}{ }^{\circ} t_{94} & =\left\{p_{95}, p_{133}\right\} \\
t_{94} & =\left\{p_{96}, p_{134}\right\}\end{aligned}$ & $\begin{aligned}{ }^{\circ} t_{95} & =\left\{\mathrm{p}_{91}, \mathrm{p}_{148}\right\} \\
\mathrm{t}_{95} & =\left\{\mathrm{p}_{92}, \mathrm{p}_{149}\right\}\end{aligned}$ \\
\hline $\begin{aligned}{ }^{\circ} t_{96} & =\left\{p_{93}, p_{143}\right\} \\
t_{96} & =\left\{p_{94}, p_{144}\right\}\end{aligned}$ & $\begin{aligned} \mathrm{t}_{97} & =\left\{\mathrm{p}_{98,}, \mathrm{p}_{124}\right\} \\
\mathrm{t}_{97} & =\left\{\mathrm{p}_{97}, \mathrm{p}_{125}\right\}\end{aligned}$ & $\begin{aligned} \cdot \mathrm{t}_{98} & =\left\{\mathrm{p}_{100}, \mathrm{p}_{129}\right\} \\
\mathrm{t}_{98} & =\left\{\mathrm{p}_{99}, \mathrm{p}_{130}\right\}\end{aligned}$ & $\begin{aligned} \mathrm{t}_{99} & =\left\{\mathrm{p}_{102}, \mathrm{p}_{149}\right\} \\
\mathrm{t}_{99} & =\left\{\mathrm{p}_{101}, \mathrm{p}_{150}\right\}\end{aligned}$ & $\begin{array}{l}\mathrm{t}_{100}=\left\{\mathrm{p}_{104}, \mathrm{p}_{144}\right\} \\
\mathrm{t}_{100}=\left\{\mathrm{p}_{103},\right. \\
\left.\mathrm{p}_{145}\right\}\end{array}$ \\
\hline $\begin{array}{l}\mathrm{t}_{101}=\left\{\mathrm{p}_{106}, \mathrm{p}_{134}\right\} \\
\mathrm{t}_{101}=\left\{\mathrm{p}_{105},\right. \\
\left.\mathrm{p}_{135}\right\}\end{array}$ & $\begin{array}{l}\mathrm{t}_{102}=\left\{\mathrm{p}_{108}, \mathrm{p}_{139}\right\} \\
\mathrm{t}_{102}=\left\{\mathrm{p}_{107},\right. \\
\left.\mathrm{p}_{140}\right\}\end{array}$ & $\begin{aligned} \cdot \mathrm{t}_{103} & =\left\{\mathrm{p}_{97}, \mathrm{p}_{134}\right\} \\
\mathrm{t}_{103} & =\left\{\mathrm{p}_{98}, \mathrm{p}_{155}\right\}\end{aligned}$ & $\begin{array}{l}\cdot \mathrm{t}_{104}=\left\{\mathrm{p}_{99}, \mathrm{p}_{159}\right\} \\
\mathrm{t}_{104}=\left\{\mathrm{p}_{100},\right. \\
\left.\mathrm{p}_{160}\right\}\end{array}$ & $\begin{array}{l}\mathrm{t}_{105}=\left\{\mathrm{p}_{103}, \mathrm{p}_{165}\right\} \\
\mathrm{t}_{105}=\left\{\mathrm{p}_{104},\right. \\
\left.\mathrm{p}_{165}\right\}\end{array}$ \\
\hline $\begin{array}{l}\mathrm{t}_{106}=\left\{\mathrm{p}_{101}, \mathrm{p}_{169}\right\} \\
\mathrm{t}_{106}=\left\{\mathrm{p}_{102},\right. \\
\left.\mathrm{p}_{170}\right\}\end{array}$ & $\begin{array}{l}\mathrm{t}_{107}=\left\{\mathrm{p}_{105}, \mathrm{p}_{174}\right\} \\
\mathrm{t}_{107}=\left\{\mathrm{p}_{106},\right. \\
\left.\mathrm{p}_{175}\right\}\end{array}$ & $\begin{array}{l}\cdot \mathrm{t}_{108}=\left\{\mathrm{p}_{107}, \mathrm{p}_{179}\right\} \\
\mathrm{t}_{108}=\left\{\mathrm{p}_{108},\right. \\
\mathrm{p}_{180\}}\end{array}$ & $\begin{array}{l}\mathrm{t}_{109}=\left\{\mathrm{p}_{110}, \mathrm{p}_{209}\right\} \\
\mathrm{t}_{109}=\left\{\mathrm{p}_{109},\right. \\
\left.\mathrm{p}_{210}\right\}\end{array}$ & $\begin{array}{l}\mathrm{t}_{110}=\left\{\mathrm{p}_{109}, \mathrm{p}_{204}\right\} \\
\mathrm{t}_{110^{*}}=\left\{\mathrm{p}_{110},\right. \\
\left.\mathrm{p}_{205}\right\}\end{array}$ \\
\hline $\begin{array}{l}\mathrm{t}_{111}=\left\{\mathrm{p}_{112}, \mathrm{p}_{199}\right\} \\
\mathrm{t}_{111}=\left\{\mathrm{p}_{111},\right. \\
\left.\mathrm{p}_{200}\right\}\end{array}$ & $\begin{array}{l}\mathrm{t}_{112}=\left\{\mathrm{p}_{114}, \mathrm{p}_{194}\right\} \\
\mathrm{t}_{112}=\left\{\mathrm{p}_{113},\right. \\
\left.\mathrm{p}_{195}\right\}\end{array}$ & $\begin{array}{l}\mathrm{t}_{113}=\left\{\mathrm{p}_{111}, \mathrm{p}_{189}\right\} \\
\mathrm{t}_{113}=\left\{\mathrm{p}_{112},\right. \\
\left.\mathrm{p}_{190\}}\right\}\end{array}$ & $\begin{array}{l}\mathrm{t}_{114}=\left\{\mathrm{p}_{113}, \mathrm{p}_{184}\right\} \\
\mathrm{t}_{114}=\left\{\mathrm{p}_{114},\right. \\
\left.\mathrm{p}_{185}\right\}\end{array}$ & $\begin{array}{l}\mathrm{t}_{115}=\left\{\mathrm{p}_{116}, \mathrm{p}_{214}\right\} \\
\mathrm{t}_{115}=\left\{\mathrm{p}_{115},\right. \\
\left.\mathrm{p}_{215}\right\}\end{array}$ \\
\hline $\begin{array}{l}\mathrm{t}_{116}=\left\{\mathrm{p}_{115}, \mathrm{p}_{219}\right\} \\
\mathrm{t}_{116}=\left\{\mathrm{p}_{116},\right. \\
\left.\mathrm{p}_{220}\right\}\end{array}$ & $\begin{array}{l}\mathrm{t}_{117}=\left\{\mathrm{p}_{118}, \mathrm{p}_{239}\right\} \\
\mathrm{t}_{117}=\left\{\mathrm{p}_{117},\right. \\
\left.\mathrm{p}_{240}\right\}\end{array}$ & $\begin{aligned} \mathrm{t}_{118} & =\left\{\mathrm{p}_{120}, \mathrm{p}_{234}\right\} \\
\mathrm{t}_{118} & =\left\{\mathrm{p}_{119}, \mathrm{p}_{235}\right.\end{aligned}$ & $\begin{array}{l}\mathrm{t}_{119}=\left\{\mathrm{p}_{117}, \mathrm{p}_{229}\right\} \\
\mathrm{t}_{119}=\left\{\mathrm{p}_{118},\right. \\
\left.\mathrm{p}_{230}\right\}\end{array}$ & $\begin{array}{l}\mathrm{t}_{120}=\left\{\mathrm{p}_{119}, \mathrm{p}_{224}\right\} \\
\mathrm{t}_{120^{\circ}}=\left\{\mathrm{p}_{120},\right. \\
\left.\mathrm{p}_{225}\right\}\end{array}$ \\
\hline
\end{tabular}


Let $\pi=\left\{\pi_{1}, \pi_{2}, \pi_{3}, \ldots, \pi_{84}\right\}$ be the partition of the set of places of $S_{5}$. where $\pi_{1}=\left\{\mathrm{p}_{121}, \mathrm{p}_{122}, \mathrm{p}_{123}, \mathrm{p}_{124}, \mathrm{p}_{125}\right\}, \pi_{2}=\left\{\mathrm{p}_{126}, \mathrm{p}_{127}, \mathrm{p}_{128}, \mathrm{p}_{129}, \mathrm{p}_{130}\right\}, \pi_{3}=\left\{\mathrm{p}_{131}, \mathrm{p}_{132}, \mathrm{p}_{133}, \mathrm{p}_{134}, \mathrm{p}_{135}\right\}$, $\pi_{4}=\left\{\mathrm{p}_{136}, \mathrm{p}_{137}, \mathrm{p}_{138}, \mathrm{p}_{139}, \mathrm{p}_{140}\right\}, \pi_{5}=\left\{\mathrm{p}_{141}, \mathrm{p}_{142}, \mathrm{p}_{143}, \mathrm{p}_{144}, \mathrm{p}_{145}\right\}, \pi_{6}=\left\{\mathrm{p}_{146}, \mathrm{p}_{147}, \mathrm{p}_{148}, \mathrm{p}_{149}, \mathrm{p}_{150}\right\}$, $\pi_{7}=\left\{\mathrm{p}_{151}, \mathrm{p}_{152}, \mathrm{p}_{153}, \mathrm{p}_{154}, \mathrm{p}_{155}\right\}, \pi_{8}=\left\{\mathrm{p}_{156}, \mathrm{p}_{157}, \mathrm{p}_{158}, \mathrm{p}_{159}, \mathrm{p}_{160}\right\}, \pi_{9}=\left\{\mathrm{p}_{161}, \mathrm{p}_{162}, \mathrm{p}_{163}, \mathrm{p}_{164}, \mathrm{p}_{165}\right\}$, $\pi_{10}=\left\{\mathrm{p}_{166}, \mathrm{p}_{167}, \mathrm{p}_{168}, \mathrm{p}_{169}, \mathrm{p}_{170}\right\}, \pi_{11}=\left\{\mathrm{p}_{171}, \mathrm{p}_{172}, \mathrm{p}_{173}, \mathrm{p}_{174}, \mathrm{p}_{175}\right\}, \pi_{12}=\left\{\mathrm{p}_{176}, \mathrm{p}_{177}, \mathrm{p}_{178}, \mathrm{p}_{179}, \mathrm{p}_{180}\right\}$, $\pi_{13}=\left\{\mathrm{p}_{181}, \mathrm{p}_{182}, \mathrm{p}_{183}, \mathrm{p}_{184}, \mathrm{p}_{185}\right\}, \pi_{14}=\left\{\mathrm{p}_{186}, \mathrm{p}_{187}, \mathrm{p}_{188}, \mathrm{p}_{189}, \mathrm{p}_{190}\right\}, \pi_{15}=\left\{\mathrm{p}_{191}, \mathrm{p}_{192}, \mathrm{p}_{193}, \mathrm{p}_{194}, \mathrm{p}_{195}\right\}$, $\pi_{16}=\left\{\mathrm{p}_{196}, \mathrm{p}_{197}, \mathrm{p}_{198}, \mathrm{p}_{199}, \mathrm{p}_{200}\right\}, \pi_{17}=\left\{\mathrm{p}_{201}, \mathrm{p}_{202}, \mathrm{p}_{203}, \mathrm{p}_{204}, \mathrm{p}_{205}\right\}, \pi_{18}=\left\{\mathrm{p}_{206}, \mathrm{p}_{207}, \mathrm{p}_{208}, \mathrm{p}_{209}, \mathrm{p}_{210}\right\}$, $\pi_{19}=\left\{\mathrm{p}_{211}, \mathrm{p}_{212}, \mathrm{p}_{213}, \mathrm{p}_{214}, \mathrm{p}_{215}\right\}, \pi_{20}=\left\{\mathrm{p}_{216}, \mathrm{p}_{217}, \mathrm{p}_{218}, \mathrm{p}_{219}, \mathrm{p}_{220}\right\}, \pi_{21}=\left\{\mathrm{p}_{221}, \mathrm{p}_{222}, \mathrm{p}_{223}, \mathrm{p}_{224}, \mathrm{p}_{225}\right\}$, $\pi_{22}=\left\{\mathrm{p}_{226}, \mathrm{p}_{227}, \mathrm{p}_{228}, \mathrm{p}_{229}, \mathrm{p}_{230}\right\}, \pi_{23}=\left\{\mathrm{p}_{231}, \mathrm{p}_{232}, \mathrm{p}_{233}, \mathrm{p}_{234}, \mathrm{p}_{235}\right\}, \pi_{24}=\left\{\mathrm{p}_{236}, \mathrm{p}_{237}, \mathrm{p}_{238}, \mathrm{p}_{239}, \mathrm{p}_{240}\right\}$, $\pi_{25}=\left\{\mathrm{p}_{1}, \mathrm{p}_{2}\right\}, \quad \pi_{26}=\left\{\mathrm{p}_{3} . \mathrm{p}_{4}\right\}, \quad \pi_{27}=\left\{\mathrm{p}_{5}, \mathrm{p}_{6}\right\}, \quad \pi_{28}=\left\{\mathrm{p}_{7}, \mathrm{p}_{8}\right\}, \quad \pi_{29}=\left\{\mathrm{p}_{9}, \mathrm{p}_{10}\right\}, \pi_{30}=\left\{\mathrm{p}_{11}, \mathrm{p}_{12}\right\}$, $\pi_{31}=\left\{\mathrm{p}_{13}, \mathrm{p}_{14}\right\}, \pi_{32}=\left\{\mathrm{p}_{15}, \mathrm{p}_{16}\right\}, \pi_{33}=\left\{\mathrm{p}_{17}, \mathrm{p}_{18}\right\}, \pi_{34}=\left\{\mathrm{p}_{19}, \mathrm{p}_{20}\right\}, \pi_{35}=\left\{\mathrm{p}_{21}, \mathrm{p}_{22}\right\}, \pi_{36}=\left\{\mathrm{p}_{23}, \mathrm{p}_{24}\right\}$, $\pi_{37}=\left\{\mathrm{p}_{25}, \mathrm{p}_{26}\right\}, \pi_{38}=\left\{\mathrm{p}_{27}, \mathrm{p}_{28}\right\}, \pi_{39}=\left\{\mathrm{p}_{29}, \mathrm{p}_{30}\right\}, \pi_{40}=\left\{\mathrm{p}_{31}, \mathrm{p}_{32}\right\}, \pi_{41}=\left\{\mathrm{p}_{33}, \mathrm{p}_{34}\right\}, \pi_{42}=\left\{\mathrm{p}_{35}, \mathrm{p}_{36}\right\}$, $\pi_{43}=\left\{\mathrm{p}_{37}, \mathrm{p}_{38}\right\}, \pi_{44}=\left\{\mathrm{p}_{39}, \mathrm{p}_{40}\right\}, \pi_{45}=\left\{\mathrm{p}_{41}, \mathrm{p}_{42}\right\}, \pi_{46}=\left\{\mathrm{p}_{43}, \mathrm{p}_{44}\right\}, \pi_{47}=\left\{\mathrm{p}_{45}, \mathrm{p}_{46}\right\}, \pi_{48}=\left\{\mathrm{p}_{47}, \mathrm{p}_{48}\right\}$, $\pi_{49}=\left\{\mathrm{p}_{49}, \mathrm{p}_{50}\right\}, \pi_{50}=\left\{\mathrm{p}_{51}, \mathrm{p}_{52}\right\}, \pi_{51}=\left\{\mathrm{p}_{53}, \mathrm{p}_{54}\right\}, \pi_{52}=\left\{\mathrm{p}_{55}, \mathrm{p}_{56}\right\}, \pi_{53}=\left\{\mathrm{p}_{57}, \mathrm{p}_{58}\right\}, \pi_{54}=\left\{\mathrm{p}_{59}, \mathrm{p}_{60}\right\}$, $\pi_{55}=\left\{\mathrm{p}_{61}, \mathrm{p}_{62}\right\}, \pi_{56}=\left\{\mathrm{p}_{63}, \mathrm{p}_{64}\right\}, \pi_{57}=\left\{\mathrm{p}_{65}, \mathrm{p}_{66}\right\}, \pi_{58}=\left\{\mathrm{p}_{67}, \mathrm{p}_{68}\right\}, \pi_{59}=\left\{\mathrm{p}_{69}, \mathrm{p}_{70}\right\}, \pi_{60}=\left\{\mathrm{p}_{71}, \mathrm{p}_{72}\right\}$, $\pi_{61}=\left\{\mathrm{p}_{73}, \mathrm{p}_{74}\right\}, \pi_{62}=\left\{\mathrm{p}_{75}, \mathrm{p}_{76}\right\}, \pi_{63}=\left\{\mathrm{p}_{77}, \mathrm{p}_{78}\right\}, \pi_{64}=\left\{\mathrm{p}_{79}, \mathrm{p}_{80}\right\}, \pi_{65}=\left\{\mathrm{p}_{81}, \mathrm{p}_{82}\right\}, \pi_{66}=\left\{\mathrm{p}_{83}, \mathrm{p}_{84}\right\}$, $\pi_{67}=\left\{\mathrm{p}_{85}, \mathrm{p}_{86}\right\}, \pi_{68}=\left\{\mathrm{p}_{87}, \mathrm{p}_{88}\right\}, \pi_{69}=\left\{\mathrm{p}_{89}, \mathrm{p}_{90}\right\}, \pi_{70}=\left\{\mathrm{p}_{91}, \mathrm{p}_{92}\right\}, \pi_{71}=\left\{\mathrm{p}_{93}, \mathrm{p}_{94}\right\}, \pi_{72}=\left\{\mathrm{p}_{95}, \mathrm{p}_{96}\right\}$, $\pi_{73}=\left\{\mathrm{p}_{97}, \mathrm{p}_{98}\right\}, \pi_{74}=\left\{\mathrm{p}_{99}, \mathrm{p}_{100}\right\}, \pi_{75}=\left\{\mathrm{p}_{101}, \mathrm{p}_{102}\right\}, \pi_{76}=\left\{\mathrm{p}_{103}, \mathrm{p}_{104}\right\}, \pi_{77}=\left\{\mathrm{p}_{105}, \mathrm{p}_{106}\right\}$,

$\pi_{78}=\left\{\mathrm{p}_{107}, \mathrm{p}_{108}\right\}, \pi_{79}=\left\{\mathrm{p}_{109}, \mathrm{p}_{110}\right\}, \pi_{80}=\left\{\mathrm{p}_{111}, \mathrm{p}_{112}\right\}, \pi_{81}=\left\{\mathrm{p}_{113}, \mathrm{p}_{114}\right\}, \pi_{82}=\left\{\mathrm{p}_{115}, \mathrm{p}_{116}\right\}$, $\pi_{83}=\left\{\mathrm{p}_{117}, \mathrm{p}_{118}\right\}, \pi_{84}=\left\{\mathrm{p}_{119}, \mathrm{p}_{120}\right\}$

The above $\operatorname{ACPN}\left(S: S_{5}\right)$ is clearly a marked graph. This $\operatorname{ACPN}\left(S: S_{5}\right)$ has a subset of places which are both siphon and trap such that the input transitions equal the output transitions and both of them equal to the set of all transitions of the marked graph and hence that the underlying directed graph of this marked graph is Hamiltonian. Since there exists a partition for a place set $P$ such that each block in the partition of the set of places of the marked graph is both siphon and trap, the underlying directed graph for $\operatorname{ACPN}\left(S: S_{5}\right)$ is Eulerian.

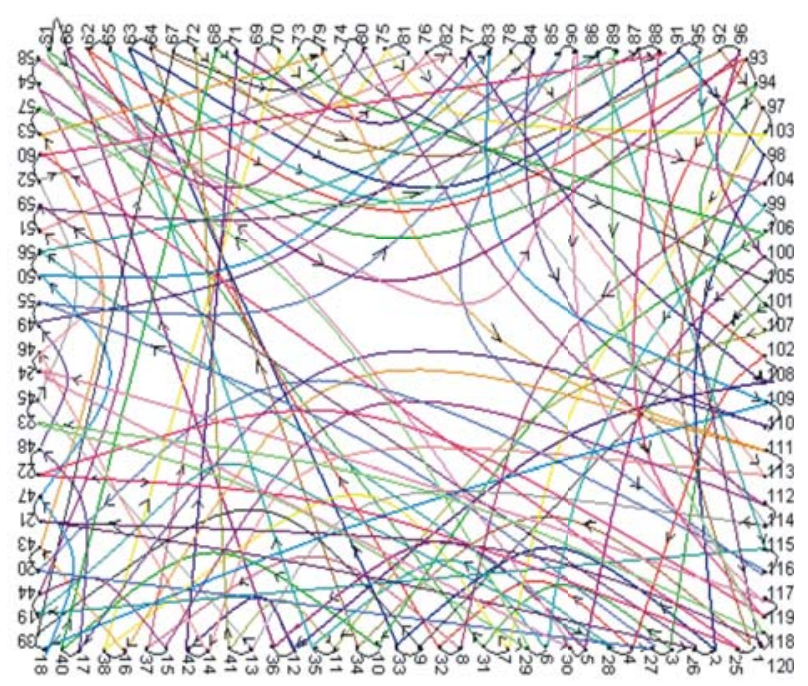

Fig .4 The underlying directed graph for the algebraic conservative Petri net given in example 3.6

\section{CONCLUSION}

In this paper we introduced a new sub class of Petri nets called algebraic conservative Petri nets (ACPN) for a given symmetric group $S_{n}$. We proved that the resulting Petri net (ACPN) is a marked graph . In particular, for the groups $S_{3}$ and $S_{5}$, we show that each of the ACPN associated with these groups has a subset of places which are both siphon and trap such that the input transitions equal the output transitions and both of them equal to the set of all transitions of these algebraic conservative Petri nets and hence that the underlying directed graphs of these algebraic conservative Petri nets are Hamiltonian. Also we shown that the algebraic conservative Petri nets associated with $\mathrm{S}_{3}$ and $\mathrm{S}_{5}$ has decompositions

$$
\pi=\left\{\pi_{1}, \pi_{2}, \pi_{3}, \pi_{4}, \pi_{5}\right\} \text { and } \pi=\left\{\pi_{1}, \pi_{2}, \pi_{3}, \pi_{4}, \ldots, \pi_{84}\right\}
$$
respectively, for the sets of places such that each block $\pi$ i is both siphon and trap and hence the underlying directed graphs of these algebraic conservative Petri nets associated with $S_{3}$ and $S_{5}$ are Eulerian.

\section{REFERENCES}

[1] Murata, T., Petri nets, Properties, Analysis and Applications, Proceedings of IEEE, 77, 541-580. (1989).

[2] Peterson J.L., Petrinet theory and the modeling of systems, Prentice Hall, Englewood Cliffs, New Jersy (1981). 
[3] Rajeswari, R and K. Thirusangu, Marked Graphs and Symmetric Groups, Proceedings of International Conference on Trends in Information Sciences and Computing, Sathyabama University, Chennai, 428-432, (2007)

[4] Thirusangu, $\mathrm{K}$ and Rangarajan, $\mathrm{K}$, Marked Graphs and Hamiltonian Graphs, Micro Electronics and Reliability, 37, 1243-1250 (1997).

[5] Thirusangu, $\mathrm{K}$ and Rangarajan, $\mathrm{K}$, Marked Graphs and Eulerian Graphs, Micro Electronics and Reliability, 37, No:2, 225-235(1997)

[6] Thirusangu, K, Ranganayakulu,D and Rangarajan, K, Marked Graph and P-groups, Acta Ciencia Indica, Vol. XXVIIM, No.3, 321 (2001).
[7] Witte D. Cayley digraphs of Prime Power Order are Hamiltonian, Journal of Combinatorial Theory, Series, B40, 107-112(1986).

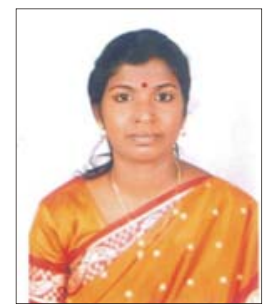

Ms. Rajeswari .R - Faculty, Department of Mathematics, Sathyabama University. She has 6 years of academic experience. She has presented 4 papers in national and international conferences. Her area of research is Algebraic Petri Nets. 